\title{
Testing Inverse Problems : a direct or an indirect problem?
}

\author{
Béatrice Laurent, Jean-Michel Loubes and Clément Marteau
}

\begin{abstract}
In this paper, we consider ill-posed inverse problems models $Y=T f+\epsilon \xi$ where $T$ denotes a compact operator, $\epsilon$ a noise level, $\xi$ a Gaussian white noise and $f$ the function of interest. Recently, minimax rates of testing in such models have been obtained in various situations, both from asymptotic and non-asymptotic point of views. Nevertheless, it seems necessary to propose tests strategies attaining theses rates, being easy to implement and robust with respect to the characteristics of the operator. In particular, we prove that the inversion of the operator is not always necessary. This result provides interesting perspectives, for instance in the specific cases where the operator is unknown or difficult to handle.
\end{abstract}

Keywords: Test, Inverse Problems

Subject Class. MSC-2000 : 62G05, 62K20

\section{Introduction}

Inverse problems have been extensively studied over the past decades. They provide a non-trivial generalization of classical statistical models and are at the core of several application problems. In this paper, we consider the model

$$
Y=T f+\sigma \xi
$$

where $T: \mathcal{X} \rightarrow \mathcal{Y}$ denotes a compact operator, $\mathcal{X}$ and $\mathcal{Y}$ Hilbert spaces, $\sigma$ a noise level and $\xi$ a Gaussian white noise.

In this framework, the most common issues mainly concern the estimation of the parameter of interest $f$, with either parametric or non parametric technics. Optimality with respect to a particular loss function have been achieved and some authors have built adaptive methods leading to general oracle inequalities. Many methods have been 
considered, representing the most trends in statistic estimation methods (kernel methods, model selection, projection onto specific bases).

Actually one of the main difference between direct and indirect problems comes from the fact that two spaces are at hand: the space of the observations $\mathcal{Y}$ and the space where the function will be estimated, namely $\mathcal{X}$, the operator mapping one space into another, $T: \mathcal{X} \rightarrow \mathcal{Y}$. Hence to build a statistical procedure, a choice must be made which will determine the whole methodology. This question is at the core of the inverse problem structure and is encountered in many cases. When trying to build basis well adapted to the operator, two strategies can be chosen, either expanding the function onto a wavelet basis of the space $\mathcal{X}$ and taking the image of the basis by the operator as stated in [6], or expanding the image of the function onto a wavelet basis of $\mathcal{Y}$ and looking at the image of the basis by the inverse of the operator, studied in [1]. For the estimation problem with model selection theory, estimators can be obtained either by considering sieves on $\left(Y_{m}\right)_{m} \subset \mathcal{Y}$ with their counterpart $X_{m}:=T^{\star} Y_{m} \subset \mathcal{X}$ or sieves on $\left(X_{m}\right)_{m} \subset \mathcal{X}$ and their image $Y_{m}:=T X_{m} \subset \mathcal{Y}$ (see for instance in $[13,12]$ ).

Signal detection for inverse problem has received little attention. If some particular cases such as convolution problems have been widely investigated (see [4] or [7] for general references), the general case of tests for inverse problem have only been tackled very recently. We refer to [8], [10] for a complete asymptotical and non asymptotical theory. The previous dilemma concerning the choice of the space where to perform the study is here also crucial. Indeed, two methods are at hand : the first one consists in performing tests on the functional space $\mathcal{X}$ which implies inverting the operator, while the second method is to build a test directly on the observations in the space $\mathcal{Y}$, which involves considering an hypothesis on the image of the unknown function. Such issues have been tackled for different alternatives in [3] or [7].

It is obvious that from a practical point of view, for known operators, testing directly the data has the advantage to be very easy to use, requiring few computations. Indeed, since $T f$ follows a direct regression model, all well known testing procedures may apply. In this paper, we will show that in many cases, considering the problem as a direct problem often leads to very interesting testing performances. Hence depending on the difficulty of the inverse problem and on the set of assumptions on the function to be detected (sparse conditions or smoothness conditions), we prove that the specific treatment devoted to inverse problem which includes an underlying inversion of the operator, may worsen the detection strategy. For each situation, we also highlight the cases where the direct strategy fails and were a specific test for inverse problem should be preferred. Deviations from an assumption on the function may be more natural to consider rather than assumption on its image by the operator, $T f$ but since we consider signal detection, i.e tests on the nullity of the function, both assumptions can be investigated. 
The paper falls into the following parts. Section 2 defines precisely the criterion chosen to describe the optimality of the tests. Section 3 is devoted to testing issues where the signal is characterized by smoothness constraints. In Section 4, we consider finite dimensional models: we present and develop some tools that will be used to prove the results of Section 3. Then, some simulation results are gathered in Section 5. A general discussion is displayed in Section 6 while the proofs are postponed to Section 7.

\section{Signal detection for inverse problems : two possible frameworks}

We consider in this paper signal detection of a function $f$ observed in the following framework. Let $T$ a linear operator on an Hilbert space $\mathcal{X}$ with inner product (.,.), and consider an unknown function $f$ observed from indirect observations in a Gaussian white noise model

$$
Y(g)=(T f, g)+\sigma \epsilon(g), g \in H
$$

where $\epsilon(g)$ is a centered Gaussian variable with variance $\|g\|^{2}:=(g, g)$. The operator $T$ is supposed to be compact. Then it admits a singular value decomposition (SVD) $\left(b_{j}, \psi_{j}, \phi_{j}\right)_{j \geq 1}$ in the sense that

$$
T \phi_{j}=b_{j} \psi_{j}, \quad T^{*} \psi_{j}=b_{j} \phi_{j} \forall j \in \mathbb{N}^{\star},
$$

where $T^{*}$ denotes the adjoint operator of $T$. Considering the observations $\left(Y\left(\psi_{j}\right)\right)_{j \in \mathbb{N}^{*}}$, Model (1) becomes

$$
Y_{j}=b_{j} \theta_{j}+\sigma \epsilon_{j}=\nu_{j}+\sigma \epsilon_{j}, \forall j \geq 1,
$$

with $Y_{j}=Y\left(\psi_{j}\right), \epsilon_{j}=\epsilon\left(\psi_{j}\right),\left(T f, \psi_{j}\right)=b_{j} \theta_{j}=\nu_{j}$ and $\theta_{j}=\left(f, \phi_{j}\right)$. Hence, inference on the sequence $\theta=\left(\theta_{j}\right)_{j \in \mathbb{N}^{\star}}$ provides the same results for the function $f$.

In order to measure the testing difficulty of a given model, we will consider the minimax point of view developed in the series of paper due to Ingster [9]. Let $\mathcal{G}$ be some subset of an Hilbert space and $g \in \mathcal{G}$ a function of interest. We consider the minimal radius $\rho$ for which the problem of testing " $g=0$ " against the alternative " $g \in \mathcal{G}$ and $\|g\| \geq \rho "$ with prescribed probabilities of errors is possible. Of course, the smaller the radius $\rho$, the better will be the test.

More precisely, a test is a decision rule $\Phi$ with value in $\{0,1\}$. By convention, we accept a null hypothesis $H_{0}$ when $\Phi=0$ and we reject otherwise. Let $\alpha \in(0,1)$ fixed, a decision rule $\Phi$ is a level- $\alpha$ test if $P_{H_{0}}(\Phi=1) \leq \alpha$ for some $\alpha>0$. In this case, we often write $\Phi_{\alpha}$ instead of $\Phi$ in order to enlighten the dependence in $\alpha$. The quality of the test $\Phi_{\alpha}$ then relies on the quantity $\rho\left(\Phi_{\alpha}, \beta, \mathcal{G}\right)$ defined as

$$
\rho\left(\Phi_{\alpha}, \beta, \mathcal{G}\right)=\inf \left\{\rho>0, \sup _{g \in \mathcal{G},\|g\| \geq \rho} P_{g}\left(\Phi_{\alpha}=0\right) \leq \beta\right\},
$$


for some fixed $\beta$. The minimax rate of testing on $\mathcal{G}$ is defined as the smallest possible rate on $\mathcal{G}$, namely

$$
\rho(\mathcal{G}, \alpha, \beta):=\inf _{\Phi_{\alpha}} \rho\left(\Phi_{\alpha}, \beta, \mathcal{G}\right)
$$

where the infimum is taken over all possible level- $\alpha$ testing procedures.

A testing procedure $\Phi_{\alpha}$ is said to be minimax for $H_{0}: g=0$ on $\mathcal{G}$ if there exists a constant $C \geq 1$ such that

$$
\sup _{g \in \mathcal{G},\|g\| \geq C \rho(\mathcal{G}, \alpha, \beta)} P_{g}\left(\Phi_{\alpha}=0\right) \leq \beta .
$$

Let $H_{0}^{I P}$ be the null hypothesis corresponding to inference on the function $f$ or the corresponding coefficients, namely

$$
H_{0}^{I P}: \quad f=0
$$

associated with the alternative $H_{1}^{I P}:\|f\| \geq \rho, f \in \mathcal{F}$ for some $\mathcal{F} \subset \mathcal{X}$. The corresponding rates of testing have been computed very recently in [8] or [10] and different testing procedures have been proposed. We may alternatively consider the hypothesis image of the assumption $H_{0}^{I P}$ by the operator $T$

$$
H_{0}^{D P}: \quad T f=0,
$$

with the alternative $H_{1}^{D P}:\|T f\| \geq \rho, T f \in T \mathcal{F}$, where $T \mathcal{F}$ denotes the image of $\mathcal{F}$ by the operator $T$. Since the operator is known, note that the assumption $H_{0}^{D P}$ is completely specified. In this case the model (3) may be viewed as a direct observation model. Previous rates have been computed for the different sets of assumptions that we will consider, conditions on the number of non zeroes coefficients or regularity assumptions. For more details, we refer to [9], [11] or [2] for a non-asymptotic point of view.

It seems clear that both assumptions $T f=0$ and $f=0$ are equivalent since the operator $T$ is injective. So $H_{0}^{I P}$ and $H_{0}^{D P}$ are two ways of rephrasing the same question. Nevertheless, remark that the associated alternatives and rates of convergence strongly differ. In some sense, the inversion of the operator may introduce an additional difficulty. Hence, a test minimax for $H_{0}^{D P}$ on $T \mathcal{F}$ is not necessary minimax for $H_{0}^{I P}$ on $\mathcal{F}$. The same remark holds for the reverse. Nevertheless, we can conjecture that in some specific cases, these hypotheses may be in some sense exchangeable or at least that there exists some kind of hierarchy. The aim of this paper is to present these situations. More precisely, we will enlighten situations where tests procedures may be used to consider both $H_{0}^{D P}$ and $H_{0}^{I P}$ in an optimal way. For different classes of functions $\mathcal{F}$, we will point out the cases where

- every procedure $\Phi_{\alpha}$ minimax for $H_{0}^{I P}$ on $\mathcal{F}$ is also necessary minimax for $H_{0}^{D P}$ on $T \mathcal{F}$, 
- every procedure $\Phi_{\alpha}$ minimax for $H_{0}^{D P}$ on $\mathcal{T} \mathcal{F}$ is also necessary minimax for $H_{0}^{I P}$ on $\mathcal{F}$.

We will see that the answer is not so simple and depends on the spaces $\mathcal{F}$ and on the ill-posedness of the problem.

We will consider different spaces $\mathcal{F}$ in order to define the alternative to $H_{0}^{D P}$ and $H_{0}^{I P}$. Hereafter we will consider inference on the function $f=\sum_{j} \theta_{j} \psi_{j}$ or the image by the operator $T, T f=\sum_{j} b_{j} \theta_{j} \psi_{j}=\sum_{j} \nu_{j} \psi_{j}$, expressing the assumptions directly on the functions or their corresponding coefficients in the appropriate basis. Concerning the operator, conditions will be set on the sequence $\left(b_{k}\right)_{k \in \mathbb{N}^{*}}$. The decay of the eigenvalues indeed describes the difficulty of the inverse problem. We will consider two main cases:

Mildly ill-posed There exist two constants $c, C$ and an index $s>0$ such that

$$
\forall j \geq 1, \quad c j^{-s} \leq b_{j} \leq C j^{-s}
$$

Severely ill-posed There exist two constants $c, C$ and an index $\gamma>0$ such that

$$
\forall j \geq 1, \quad c \exp (-\gamma j) \leq b_{j} \leq C \exp (-\gamma j)
$$

The parameters $s$ and $\gamma$ are called index of ill-posedness of the corresponding inverse problem.

\section{$3 \quad$ Tests strategies under smoothness constraints}

In the following, the functions of interest are assumed to belong to smoothness classes determined by the decay of their coefficients in the bases $\left(\phi_{k}\right)_{k \geq 1}$ or $\left(\psi_{k}\right)_{k \geq 1}$. Several explicit rates of testing have been established following the considered spaces $(\mathcal{X}$ or $\mathcal{Y})$, the rate of decay of the coefficients, and the behavior of the sequence $\left(b_{k}\right)_{k \geq 1}$. For more details, we refer for instance to [2] in the direct case (i.e. when $T$ denotes the identity) or to [10] in an heteroscedastic setting, which is equivalent to the inverse problem modeled in $(3)$.

More precisely, let $a=\left(a_{k}\right)_{k \in \mathbb{N}^{*}}$ be a monotone non-decreasing sequence and $R>0$. In the following, we assume that the function $f$ is embedded in an ellipsoid $\mathcal{E}_{a, 2}^{\mathcal{X}}(R)$ of the form

$$
\mathcal{E}_{a, 2}^{\mathcal{X}}(R)=\left\{g \in \mathcal{X}, \sum_{j=1}^{+\infty} a_{j}^{2}\left\langle g, \phi_{j}\right\rangle^{2} \leq R^{2}\right\} .
$$

The sequence $a$ characterizes the shape of the ellipsoid. Functional sets of the form (4) are often used to model some smoothness class of functions : the choice $a_{j}=j^{s}$ for all $j \in \mathbb{N}^{\star}$ 
corresponds to a Sobolev ball with regularity $s$, namely $H^{s}(R)$ while $a_{j}=\exp (s j)$ for all $j \in \mathbb{N}^{\star}$ entails that the function belongs to an analytic class of function with parameter $s$. We can also define the analogue of $\mathcal{E}_{a, 2}^{\mathcal{X}}(R)$ on $\mathcal{Y}$, denoted in the following by $\mathcal{E}_{c, 2}^{\mathcal{Y}}(R)$, for a given sequence $c$.

Imposing an ellipsoid type constraint on the function $f$ entails also an ellipsoid constraint on $T f$. Indeed, using Model (3), we get that $T f \in \mathcal{E}_{c, 2}^{\mathcal{Y}}(R)$ as soon as $f \in \mathcal{E}_{a, 2}^{\mathcal{X}}(R)$, for $c=\left(c_{k}\right)_{k \in \mathbb{N}}=\left(a_{k} b_{k}^{-1}\right)_{k \in \mathbb{N}}$. We point out that the operator regularizes the function and for such a smoothness assumption, function $T f$ is smoother than $f$.

On the one hand, the rates for the inverse hypothesis $H_{0}^{I P}$ highly depend on the ill-posedness of the inverse problem and the different types of ellipsoids. They are given in [10] and recalled in Table 1 below. In the following, we write $\mu_{k} \sim \nu_{k}$ if there exists two constants $c_{1}$ and $c_{2}$ such that for all $k \geq 1, c_{1} \leq \mu_{k} / \nu_{k} \leq c_{2}$. The Table 1 presents the minimax rates of testing over the ellipsoids $\mathcal{E}_{a, 2}^{\mathcal{X}}(R)$ with respect to the $l_{2}$ norm. We consider various behaviours for the sequences $\left(a_{k}\right)_{k \in \mathbb{N}^{*}}$ and $\left(b_{k}\right)_{k \in \mathbb{N}^{*}}$. For each case, we give $f(\sigma)$ such that for all $0<\sigma<1, C_{1}(\alpha, \beta) f(\sigma) \leq \rho^{2}\left(\mathcal{E}_{a, 2}^{\mathcal{X}}(R), \alpha, \beta\right) \leq C_{2}(\alpha, \beta) f(\sigma)$ where $C_{1}(\alpha, \beta)$ and $C_{2}(\alpha, \beta)$ denote positive constants independent of $\sigma$.

\begin{tabular}{lcc}
\hline & $\begin{array}{c}\text { Mildly ill-posed } \\
b_{k} \sim k^{-t}\end{array}$ & $\begin{array}{c}\text { Severely ill-posed } \\
b_{k} \sim \exp (-\gamma k)\end{array}$ \\
\hline$a_{k} \sim k^{s}$ & $\sigma^{2 s+2 t+1 / 2}$ & $\left(\log \left(\sigma^{-2}\right)\right)^{-2 s}$ \\
\hline$a_{k} \sim \exp \left(\nu k^{s}\right)$ & $\sigma^{2}\left(\log \left(\sigma^{-2}\right)\right)^{(2 t+1 / 2) / s}$ & $e^{-2 \nu \tilde{D}^{s}}(s<1)$ \\
\hline
\end{tabular}

Table 1: Minimax rates of testing in the indirect case.

Here $\tilde{D}$ denotes the integer part of the solution of $2 \nu D^{s}+2 \gamma D=\log \left(\sigma^{-2}\right)$ and $t, s, \nu, \gamma$ are positive constants.

On the other hand, in [2], the rates for the direct hypothesis $H_{0}^{D P}$ are obtained under the ellipsoid condition $T f \in \mathcal{E}_{c, 2}^{\mathcal{Y}}(R)$ and are presented in Table 2 below.

\begin{tabular}{lc}
\hline$c_{k} \sim k^{u}$ & $\sigma^{\frac{4 u}{2 u+1 / 2}}$ \\
\hline$c_{k} \sim \exp \left(\alpha k^{u}\right)$ & $\sigma^{2}\left(\log \left(\sigma^{-2}\right)\right)^{1 / 2 u}$ \\
\hline
\end{tabular}

Table 2: Minimax rates of testing in the direct case. 
The terms $u, \alpha$ are positive constants. Test strategies in this framework are based on the following theorem.

Theorem 3.1 Let $\left(Y_{j}\right)_{j \geq 1}$ a sequence obeying to model (3). Let $\alpha, \beta \in(0,1)$ be fixed. Let $\mathcal{E}_{a, 2}^{\mathcal{X}}(R)$ the ellipsoid defined in (4). We assume that $0<\sigma<1$. Then, in the four cases displayed in Table 1, we have

- Every level- $\alpha$ test minimax for $H_{0}^{D P}$ on $\mathcal{E}_{c, 2}^{\mathcal{Y}}(R)$ is also minimax for $H_{0}^{I P}$ on $\mathcal{E}_{a, 2}^{\mathcal{X}}(R)$,

- There exist level- $\alpha$ tests minimax for $H_{0}^{I P}$ on $\mathcal{E}_{a, 2}^{\mathcal{X}}(R)$ but not for $H_{0}^{D P}$ on $\mathcal{E}_{c, 2}^{\mathcal{Y}}(R)$,

where for all $k \geq 1, c_{k}=a_{k} b_{k}^{-1}$.

Note that under ellipsoid constraint, previous results hold both for mildly and severely ill-posed problems. Hence the conclusion of this theorem is that testing in the space of observations should be preferred rather than building specific tests designed for inverse problem which will not improve the rates and will introduce additional difficulties. Remark that we do not claim that all the procedure minimax for $H_{0}^{I P}$ will necessary fail for testing $H_{0}^{D P}$, but rather that an additional study seems necessary.

A part of the proof of Theorem 3.1 is based on Lemma 3.2 below and is postponed to Section 7. This lemma provides, under ellipsoid constraint, an embedding on the deviation balls for $T f$ provided that $f$ is bounded away from zero.

Lemma 3.2 Let $\gamma_{\sigma}$ a positive sequence such that $\gamma_{\sigma} \rightarrow 0$ as $\sigma \rightarrow 0$. The following embedding holds:

$$
\left\{f \in \mathcal{E}_{a, 2}^{\mathcal{X}}(R),\|f\|^{2} \geq \gamma_{\sigma}\right\} \subset\left\{f \in \mathcal{E}_{a, 2}^{\mathcal{X}}(R),\|T f\|^{2} \geq(1-c) \mu_{\sigma}\right\},
$$

where $c \in] 0,1\left[, \mu_{\sigma}=b_{m(\sigma)}^{2} \gamma_{\sigma}\right.$ and $m(\sigma)$ is such that $R^{2} a_{m(\sigma)}^{-2} \leq c \gamma_{\sigma}$.

Using Lemma 3.2, we can control the norm of $T f$ given $\|f\|^{2}$ for all $f$ belonging to $\mathcal{E}_{a, 2}^{\mathcal{X}}(R)$. Remark that the reverse is not true. Indeed, we can always construct signals such that $\|T f\|^{2}$ and $\|f\|^{2}$ are of the same order.

\section{Rate optimal strategies for finite dimensional mod- els}

The aim of this section is to present and develop some tools useful for the proofs of the results presented in the previous section. In particular, our aim is to exhibit tests that fail to be powerful simultaneously in the inverse and the direct setting (i.e. for the hypothesis $H_{0}^{D P}$ and $H_{0}^{I P}$ ). Most of the tests designed for alternatives of the 
form " $f \in \mathcal{E}_{a, 2}^{\mathcal{X}}(R),\|f\| \geq \rho "$ involve only a finite number of coefficients. These tests are in some sense biased. The key issue is thus to control this bias with respect to the rates of testing. As in classical nonparametric estimation problems, finding the best trade-off between these two quantities is at the heart of the testing approach. A good understanding of such kind of testing procedures is thus necessary.

Hence, in this section, we deal with functions having zero coefficients after a certain level. For a given $D \geq 1$, let

$$
\begin{aligned}
& H_{D}=\operatorname{span}\left\{\phi_{j}, 1 \leq j \leq D\right\}, \\
& K_{D}=\operatorname{span}\left\{\psi_{j}, 1 \leq j \leq D\right\},
\end{aligned}
$$

where $\operatorname{span}(A)$ denotes the linear space generated by $A \subset l_{2}(\mathbb{N})$. From (2), the assertion $f \in H_{D}$ is equivalent to $T f \in K_{D}$.

Assume that $f \in H_{D}$, or equivalently that $T f \in K_{D}$ which entails that the signal $f$ is concentrated on the first $D$ coefficients of the basis $\left(\phi_{k}\right)_{k \in \mathbb{N}}$. As said before in Section 2, choosing the best framework for building signal detection tests involves knowing the minimax rates of testings for the both settings : the direct and the indirect version of the observation model. On the one hand, rates of testing for the inverse problem (3) have been obtained in [10] under such assumptions. For $C_{L}(\alpha, \beta), C_{U}(\alpha, \beta)$ two positive constants, we have

$$
C_{L}(\alpha, \beta) \sigma^{2}\left(\sum_{j=1}^{D} b_{j}^{-4}\right)^{\frac{1}{2}} \leq \rho_{2}^{2}\left(H_{D}\right) \leq C_{U}(\alpha, \beta) \sigma^{2}\left(\sum_{j=1}^{D} b_{j}^{-4}\right)^{\frac{1}{2}} .
$$

On the other hand, for the direct observation model, $Y_{j}=\nu_{j}+\epsilon \xi_{j}, j \in \mathbb{N}$, rates are given in [2]. For $c_{L}(\alpha, \beta), c_{U}(\alpha, \beta)$ two positive constants, we get

$$
c_{L}(\alpha, \beta) \sigma^{2} \sqrt{D} \leq \rho_{2}^{2}\left(K_{D}\right) \leq c_{U}(\alpha, \beta) \sigma^{2} \sqrt{D} .
$$

For fixed $D$, we are here in a parametric setting, both rates of testing are of order $\sigma^{2}$. In order to compare the hypotheses $H_{0}^{D P}$ and $H_{0}^{I P}$ in the spirit of Section 3, we have to take into account the dependency with respect to the parameter $D$ of these rates. In this sense, we introduce a definition of rate-optimality which will provide a framework for potential comparisons between $H_{0}^{D P}$ and $H_{0}^{I P}$.

Definition 4.1 Assume that $Y=\left(Y_{j}\right)_{j \geq 1}$ obeys to Model (3). Let $\left.\alpha, \beta \in\right] 0,1[$. For all $D \in \mathbb{N}^{*}$, we consider a level $-\alpha$ test $\Phi_{\alpha, D}: \mathbb{P}_{f=0}\left(\Phi_{\alpha, D}=1\right) \leq \alpha$. The collection of tests $\left(\Phi_{\alpha, D}, D \geq 1\right)$ is rate-optimal for $H_{0}^{I P}$ over the sets $\left(H_{D}, D \geq 1\right)$ if the following property holds :

$$
\exists C_{\alpha, \beta}, \forall D \geq 1, \forall f \in H_{D},\|f\|^{2} \geq \sigma^{2} C_{\alpha, \beta}\left(\sum_{j=1}^{D} b_{j}^{-4}\right)^{\frac{1}{2}} \Longrightarrow \mathbb{P}_{f}\left(\Phi_{\alpha, D}=1\right) \geq 1-\beta .
$$


The collection of tests $\left(\Phi_{\alpha, D}, D \geq 1\right)$ is rate-optimal for $H_{0}^{D P}$ over the sets $\left(K_{D}, D \geq 1\right)$ if the following property holds :

$$
\exists C_{\alpha, \beta}^{\prime} \forall D \geq 1, \forall f \in H_{D},\|T f\|^{2} \geq \sigma^{2} C_{\alpha, \beta}^{\prime} \sqrt{D} \Longrightarrow \mathbb{P}_{f}\left(\Phi_{\alpha, D}=1\right) \geq 1-\beta .
$$

Following Definition 4.1, a family is rate optimal if, in some sense, it possesses a similar behaviour for all the values of $D$.

The following theorem provides a comparison for the two signal detection procedures at hand.

Theorem 4.2 Assume that $Y=\left(Y_{j}\right)_{j \geq 1}$ obeys to Model (3). Let $0<\alpha<\beta<1 / 2$.

\section{Mildly ill-posed inverse problem}

Let $\left(\Phi_{\alpha, D}\right)_{D \geq 1}$ be a collection of level- $\alpha$ tests which is rate-optimal for $H_{0}^{D P}$ over the sets $\left(K_{D}, D \geq 1\right)$ then $\left(\Phi_{\alpha, D}\right)_{D \geq 1}$ is also rate-optimal for $H_{0}^{I P}$ over the sets $\left(H_{D}, D \geq 1\right)$.

\section{Mildly or severely ill-posed inverse problem}

There exists a collection of tests $\left(\Phi_{\alpha, D}\right)_{D \geq 1}$ which is rate-optimal for $H_{0}^{I P}$ over the sets $\left(H_{D}, D \geq 1\right)$ but not for $H_{0}^{D P}$ over the sets $\left(K_{D}, D \geq 1\right)$.

\section{Severely ill-posed inverse problem}

There exists a collection of tests $\left(\Phi_{\alpha, D}\right)_{D \geq 1}$ which is rate-optimal for $H_{0}^{D P}$ over the sets $\left(K_{D}, D \geq 1\right)$ but not for $H_{0}^{I P}$ over the sets $\left(H_{D}, D \geq 1\right)$.

Previous theorem proves that the situation where the function is defined by a fixed number of coefficients differs from the case where a regularity constraint is added on its coefficients. On the one hand, when considering mildly ill-posed inverse problems, it seems clear that the both problems of testing respectively $H_{0}^{I P}$ and $H_{0}^{D P}$ are not equivalent when the function $f$ belongs to $H_{D}$. Every testing procedure that works in the direct case could be used without additional assumptions in the inverse case. The reverse is not true. On the other hand, the severely ill-posed case is not a straightforward generalization of the results obtained for polynomially decreasing eigenvalues. Indeed, in this particular setting, testing $H_{0}^{I P}$ and $H_{0}^{D P}$ seems to be two different tasks. A good test for $H_{0}^{I P}$ may fail for $H_{0}^{D P}$ and the reverse is true.

Hence, without additional assumption on the function $f$, different strategies should be used according to the considered assumption. 


\section{Simulations}

Our goal is to illustrate the difference between direct and indirect testing strategies on some simulations. In Figure 1, we focus on mildly ill-posed problems for testing the nullity of two coefficients. We compare and plot here the power of an indirect test $\Phi_{D, \alpha}^{(2)}$ and a direct test $\Phi_{D, \alpha}^{(1)}$ with same level $5 \%$.

The test $\Phi_{D, \alpha}^{(1)}$ rejects the null hypothesis $H_{0}: f=0$, if

$$
\sum_{j=1}^{D} Y_{j}^{2}>v_{D, \alpha}
$$

where $v_{D, \alpha}$ denotes the $1-\alpha$ quantile of $\sum_{j=1}^{D} Y_{j}^{2}$ under $H_{0}$. The test $\Phi_{D, \alpha}^{(2)}$ rejects the null hypothesis $H_{0}: f=0$, if

$$
\sum_{j=1}^{D} b_{j}^{-2} Y_{j}^{2}>u_{D, \alpha}
$$

where $u_{D, \alpha}$ denotes the $1-\alpha$ quantile of $\sum_{j=1}^{D} b_{j}^{-2} Y_{j}^{2}$ under $H_{0}$.

We will conduct two different simulations. First, for an alternative $f \in H_{2}$, we estimate the power of the tests $\Phi_{2, \alpha}^{(1)}$ and $\Phi_{2, \alpha}^{(2)}$, for the $Y_{j}$ 's obeying to Model (3), with on the one hand $b_{j}=j^{-1}$, and on the other hand $\left(\theta_{1}, \theta_{2}\right) \in(-5,5)^{2}$ ( and $\theta_{j}=0, \forall j \geq 2$ ).

The simulations are obtained using 10000 replications. In Figure 1, we present the estimated powers of the two tests. As expected, the direct test is powerful outside an ellipsoid while the indirect test behaves well outside a ball. Then, in Figure 2, we provide the difference between the two test powers. The comparison of the two tests enlightens that specific tests for inverse problems are outperformed by a procedure that directly tests the observations.

In a second simulation, we focus on two generic examples of goodness of fit. We consider two different alternatives $f_{1, D}$ an $f_{2, D}$ defined respectively as

$$
<f_{1, D}, \phi_{j}>=\left\{\begin{array}{ll}
C_{1} D^{1 / 4} & \text { if } j=1 \\
0 & \forall j>1
\end{array}, \quad<f_{2, D}, \phi_{j}>=\left\{\begin{array}{ll}
C_{1} D^{1+1 / 4} & \text { if } j=D \\
0 & \forall j \neq D
\end{array} .\right.\right.
$$

where $C_{1}=\sqrt{6}$. We perform these simulations for a mildly ill-posed problem : we set $b_{j}=j^{-1}$ for all $j \geq 1$.

Remark that for a given $D$, both functions $f_{1, D}$ an $f_{2, D}$ belong to $H_{D}$. We plot for different values of $D=1, \ldots, 50$ the second kind error for the direct test $\Phi_{\alpha, D}^{(1)}$ in dotted line and for the indirect test $\Phi_{\alpha, D}^{(2)}$ in straight line, with $\alpha=5 \%$. Results are displayed in 

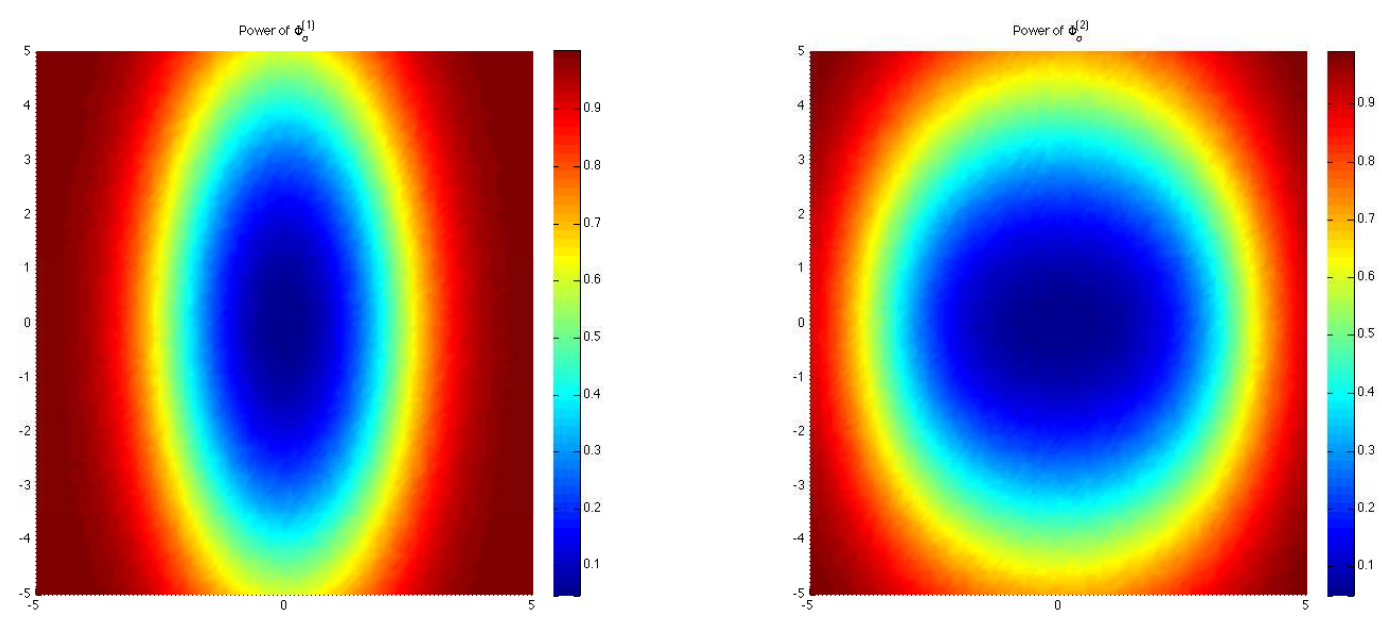

Figure 1: Power of direct and indirect tests

Figure 3. For each run, the test is conducted over 10000 replications.

For the function $f_{1, D}$, the indirect test fails in detecting the non zero coefficient while the direct test succeeds. We point out that this function corresponds to the counterexample exhibited in the proof of Theorem 4.2.

Remark also that the function $f_{2, D}$ corresponds to the theoretical case for which the direct test does not perform better than the indirect test. This statement appears also clearly on the curves since the simulated second kind errors are of the same order whatever $D$ is.

\section{Concluding Remarks}

Our aim was to be able to decide whether tests for inverse problems should be performed or if direct inference on the observations could be sufficient or even lead to better results. Note that, we only have considered tests problems for which the null hypothesis was $f=0$. Actually, this is not restrictive and the same results still hold for any goodness of fit problem of the type $f=f_{0}$. As shown in this paper, we promote the following testing strategies according to the nature of the inverse problem.

- Mildly ill-posed problems : in this situation, in most cases, tests built directly on the rough observed data, i.e in the space of the observations, outperform tests that invert the operator. Note that we have not investigated the special situation of 


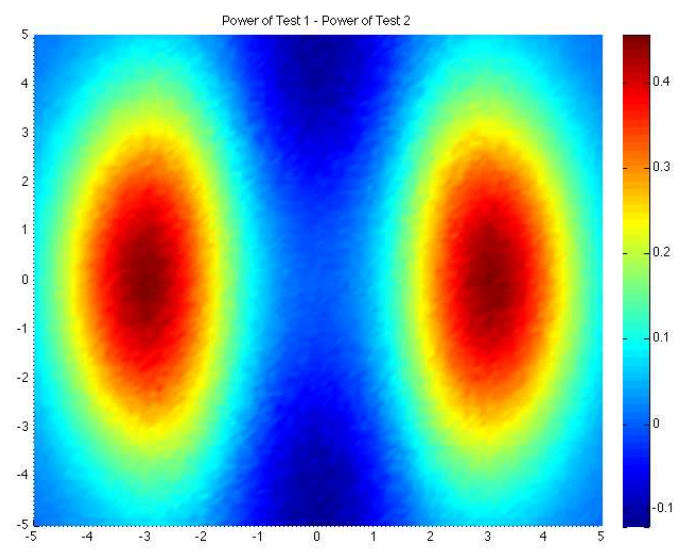

Figure 2: Comparison of direct and indirect tests

$\ell_{p}$ bodies tackled in [2] or in [10]. Indeed, for this case, minimax rates of testing are only achieved up to a logarithmic term and in this case specific tests should be designed to avoid flawed rates of testing.

- Severely ill-posed problems : for this kind of inverse problem, tests are more difficult and the situation is reversed. Indeed, in most cases, the operator changes the difficulty of the testing issues and thus a specific treatment must be addressed to the data. Only the specific frame of signal testing where the function has an ellipsoid-type regularity, enables to get rid of the inverse problem settings and to use direct testing procedure on the raw data.

Hence a large number of goodness of fit issues for inverse problems can be more efficiently solved by using tests on the direct observations, with the great advantage that a large variety of tests are available. Moreover, since only the observations are needed, as soon as the kind of inverse problem is known, tests can be designed without knowing the operator. Hence, our results enable to build tests for inverse problems with unknown operators. Such situations are encountered in [5] where the operators are unknown or partially known for instance. Nevertheless, our testing procedures in this direct case only consider alternatives of the form $\|T f\| \geq \rho$. In some cases, deviations from Assumptions $H_{0}^{I P}$ may be more natural to consider rather than applying the operator $T$.

To conclude this discussion, we point out that when it is difficult to assess which strategy should be used, we can always combine two level- $\alpha / 2$ tests respectively minimax for $H_{0}^{I P}$ and $H_{0}^{D P}$, to obtain a global minimax test. 

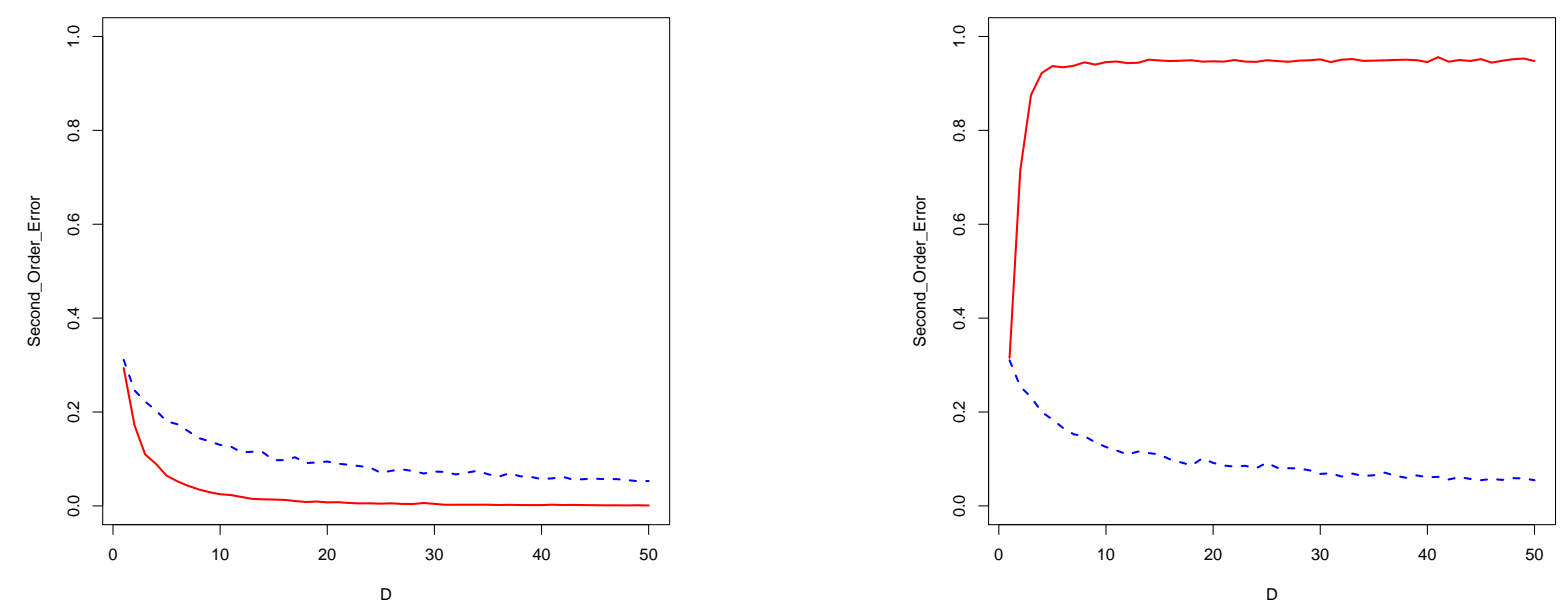

Figure 3: Second kind error for direct and indirect approaches. The dashed lines represent the second kind error for the test $\Phi_{D, \alpha}^{(1)}$ for different values of $D$ while the solid lines are associated to the test $\Phi_{D, \alpha}^{(2)}$. Results for the alternatives $f_{1, D}$ and $f_{2, D}$ introduced in (7) are respectively displayed on the left-hand and right-hand sides.

\section{Proofs}

\subsection{Technical tools}

Let us recall the following lemma which is proved in [10] (see Lemma 2).

Lemma 7.1 Let $D \in \mathbb{N}^{*}$,

$$
Z_{j}=\lambda_{j}+\sigma_{j} \epsilon_{j}, \quad 1 \leq j \leq D
$$

where $\epsilon_{1}, \ldots \epsilon_{D}$ are i.i.d. Gaussian variables with mean 0 and variance 1 . We define $\hat{T}=\sum_{j=1}^{D} Z_{j}^{2}$ and

$$
\Sigma=\sum_{j=1}^{D} \sigma_{j}^{4}+2 \sum_{j=1}^{D} \sigma_{j}^{2} \lambda_{j}^{2} .
$$

The following inequalities hold for all $x \geq 0$ :

$$
\begin{gathered}
\mathbb{P}\left(\hat{T}-\mathbb{E}(\hat{T}) \geq 2 \sqrt{\Sigma x}+2 \sup _{1 \leq j \leq D}\left(\sigma_{j}^{2}\right) x\right) \leq \exp (-x) . \\
\mathbb{P}(\hat{T}-\mathbb{E}(\hat{T}) \leq-2 \sqrt{\Sigma x}) \leq \exp (-x) .
\end{gathered}
$$


We can easily deduce from this lemma the following corollary.

\section{Corollary 7.2 Let}

$$
Y_{j}=b_{j} \theta_{j}+\sigma \epsilon_{j}, \quad j \in \mathbb{N}^{*} .
$$

Let $D \geq 1, \beta \in] 0,1\left[\right.$ and denote by $q_{1-\beta}(D)$ the $(1-\beta)$ quantile of $\sum_{j=1}^{D} b_{j}^{-2} Y_{j}^{2}$, and by $q_{1-\beta}^{\prime}(D)$ the $(1-\beta)$ quantile of $\sum_{j=1}^{D} Y_{j}^{2}$. Then, setting $x_{\beta}=\ln (1 / \beta)$

$$
\begin{gathered}
q_{1-\beta}(D) \leq \sum_{j=1}^{D} \theta_{j}^{2}+\sigma^{2} \sum_{j=1}^{D} b_{j}^{-2}+2 \sqrt{\Sigma x_{\beta}}+2 \sigma^{2} \sup _{j=1 \ldots D} b_{j}^{-2} x_{\beta} . \\
q_{1-\beta}^{\prime}(D) \leq \sum_{j=1}^{D} b_{j}^{2} \theta_{j}^{2}+\sigma^{2} D+2 \sqrt{\Sigma^{\prime} x_{\beta}}+2 \sigma^{2} x_{\beta},
\end{gathered}
$$

where

$$
\Sigma=\sigma^{4} \sum_{j=1}^{D} b_{j}^{-4}+2 \sigma^{2} \sum_{j=1}^{D} \frac{\theta_{j}^{2}}{b_{j}^{2}}, \text { and } \Sigma^{\prime}=\sigma^{4} D+2 \sigma^{2} \sum_{j=1}^{D} b_{j}^{2} \theta_{j}^{2} .
$$

Lemma 7.3 Let $\left(Y_{j}\right)_{j \geq 1}$ a sequence obeying to model (3). Let $\alpha, \beta \in(0,1)$ be fixed and $\mathcal{E}_{c, 2}^{\mathcal{Y}}(R)$ the ellipsoid introduced in Section 3. Assume that

$$
\tilde{c}_{1} e^{\alpha_{1} k^{u}} \leq c_{k} \leq \tilde{c}_{2} e^{\alpha_{2} k^{u}}, \forall k \in \mathbb{N}^{\star},
$$

where $\tilde{c}_{1}, \tilde{c}_{2}, \alpha_{1}$ and $\alpha_{2}$ denote positive constants independent of $k$. Then, there exists $\tilde{c}_{3}, \tilde{c}_{4}$ such that

$$
\tilde{c}_{3} \sigma^{2}\left(\log \left(\sigma^{-2}\right)\right)^{1 / 2 u} \leq \rho^{2}\left(\mathcal{E}_{c, 2}^{\mathcal{Y}}(R), \alpha, \beta\right) \leq \tilde{c}_{4} \sigma^{2}\left(\log \left(\sigma^{-2}\right)\right)^{1 / 2 u} .
$$

PROOF. The proof follows the same lines as in [10] (see Corollary 3). Recall from [2] or [10] that the minimax rate on $\mathcal{E}_{c, 2}^{\mathcal{Y}}(R)$ verifies

$$
\sup _{D \in J}\left(\rho_{D}^{2} \wedge R^{2} C c_{D}^{-2}\right) \leq \rho^{2}\left(\mathcal{E}_{c, 2}^{\mathcal{Y}}(R), \alpha, \beta\right) \leq \inf _{D \in J}\left(C \rho_{D}^{2}+R^{2} C c_{D}^{-2}\right),
$$

where $C$ is a positive constant depending only on $\alpha, \beta$ and $\rho_{D}^{2}=\mathcal{O}\left(\sigma^{2} \sqrt{D}\right)$ as $D \rightarrow+\infty$. In a first time, we set

$$
D_{0}=\left\lceil\left(\frac{1}{2 \alpha_{1}} \log \left(\sigma^{-2}\right)\right)^{1 / u}\right\rceil \text {. }
$$

Then

$$
\begin{aligned}
\rho^{2}\left(\mathcal{E}_{c, 2}^{\mathcal{Y}}(R), \alpha, \beta\right) & \leq C \rho_{D_{0}}^{2}+C R^{2} c_{D_{0}}^{-2} \\
& \leq C \sigma^{2}\left(\log \left(\sigma^{-2}\right)\right)^{1 / 2 u}+C \sigma^{2} \\
& \leq C \sigma^{2}\left(\log \left(\sigma^{-2}\right)\right)^{1 / 2 u}
\end{aligned}
$$


where $C$ denotes a constant independent of $\sigma$. Concerning the lower bound, we set

$$
D_{1}=\left\lfloor\left(\frac{1}{4 \alpha_{2}} \log \left(\sigma^{-2}\right)\right)^{1 / u}\right\rfloor
$$

Then

$$
\begin{aligned}
\rho^{2}\left(\mathcal{E}_{a, 2}^{\mathcal{Y}}(R), \alpha, \beta\right) & \geq \rho_{D_{1}}^{2} \wedge R^{2} C c_{D_{1}}^{-2}, \\
& \geq C \sigma^{2}\left(\log \left(\sigma^{-2}\right)\right)^{1 / 2 u} \wedge \sigma=C \sigma^{2}\left(\log \left(\sigma^{-2}\right)\right)^{1 / 2 u},
\end{aligned}
$$

for some $C>0$. This concludes the proof.

\subsection{Proof of Theorem 4.2}

For the sake of convenience, we set $\sigma=1$ in the sequel without loss of generality. Let us prove the first point of Theorem 4.2. We consider mildly ill-posed inverse problems. Let $\left(\Phi_{\alpha, D}, D \geq 1\right)$ be a collection of level- $\alpha$ tests which is rate-optimal for $H_{0}^{D P}$ on $K_{D}$. Then, for all $D \geq 1$

$$
P_{0}\left(\Phi_{\alpha, D}=1\right) \leq \alpha, \text { and } \sup _{\left\{f \in H_{D}:\|T f\|^{2} \geq C_{\alpha, \beta}^{\prime}(\sqrt{D}\}\right.} P_{f}\left(\Phi_{\alpha}=1\right) \geq 1-\beta
$$

for some constant $C_{\alpha, \beta}^{\prime}>0$. Remark that $\left(\Phi_{\alpha, D}, D \geq 1\right)$ is also a collection of level- $\alpha$ tests for $H_{0}^{I P}$. For mildly ill-posed inverse problems $\left(c j^{-s} \leq b_{j} \leq C j^{-s}\right.$ for all $\left.j \geq 1\right)$, there exists a constant $C(s)$ such that

$$
\inf _{j=1 . . D} b_{j}^{2}\left(\sum_{j=1}^{D} b_{j}^{-4}\right)^{\frac{1}{2}} \geq C(s) \sqrt{D} .
$$

Hence, for all $f \in H_{D}$ such that $\|f\|^{2}=\sum_{j=1}^{D} \theta_{j}^{2} \geq C_{\alpha, \beta}\left(\sum_{j=1}^{D} b_{j}^{-4}\right)^{\frac{1}{2}}$

$$
\|T f\|^{2}=\sum_{j=1}^{D} b_{j}^{2} \theta_{j}^{2} \geq \inf _{j=1 . . D} b_{j}^{2} \sum_{j=1}^{D} \theta_{j}^{2} \geq C(s) \times C_{\alpha, \beta}(\sqrt{D} .
$$

Since (6) holds for all constant $C_{\alpha, \beta}$ ( such that $C(s) \times C_{\alpha, \beta}\left(\geq C_{\alpha, \beta}^{\prime}\right.$, (5) holds which concludes the first part of the proof.

Let us now prove the second point of Theorem 4.2. We consider mildly or severely ill-posed inverse problems. We consider the following testing procedures : for all $D \geq 1$, let

$$
\Phi_{\alpha, D}^{I P}=\mathbf{1}_{\left\{\sum_{j=1}^{D} b_{j}^{-2} Y_{j}^{2} \geq s_{D, \alpha}\right\}}
$$


where

$$
s_{D, \alpha}=\sum_{j=1}^{D} b_{j}^{-2}+2 \sqrt{\sum_{j=1}^{D} b_{j}^{-4} x_{\alpha}}+2 \sup _{1 \leq j \leq D} b_{j}^{-2} x_{\alpha}
$$

with $x_{\alpha}=\ln (1 / \alpha)$. It is proved in [10] that for all $D \geq 1, \Phi_{\alpha, D}^{I P}$ is a level- $\alpha$ test which is rate-optimal for $H_{0}^{I P}$ on $H_{D}$. We want to show that the collection of tests $\left(\Phi_{\alpha, D}, D \geq 1\right)$ is not rate-optimal for $H_{0}^{D P}$ over the sets $\left(K_{D}, D \geq 1\right)$, i.e. that for all $C>0$, we can find $D \geq 1$ and $f \in H_{D}$ such that $\|T f\|^{2} \geq C \sqrt{D} \sigma^{2}$ and

$$
\mathbb{P}_{f}\left(\Phi_{\alpha, D}^{I P}=1\right)=\mathbb{P}_{f}\left(b_{j}^{-2} Y_{j}^{2} \geq s_{D, \alpha}\right) \leq \beta
$$

Let $q_{1-\beta}(D)$ denote the $(1-\beta)$ quantile of $\sum_{j=1}^{D} b_{j}^{-2} Y_{j}^{2}$. Inequality (14) holds if $s_{D, \alpha} \geq$ $q_{1-\beta}(D)$. Using $(10)$, with $\sigma=1$, the condition $s_{D, \alpha} \geq q_{1-\beta}(D)$ is satisfied if

$$
\sum_{j=1}^{D} \theta_{j}^{2}+2 \sqrt{\Sigma x_{\beta}} \leq 2 \sqrt{\sum_{j=1}^{D} b_{j}^{-4} x_{\alpha}}+2 \sup _{1 \leq j \leq D} b_{j}^{-2}\left(x_{\alpha}-x_{\beta}\right) .
$$

Using the inequality $\sqrt{a+b} \leq \sqrt{a}+\sqrt{b}$ for $a, b>0$, the above inequality holds if

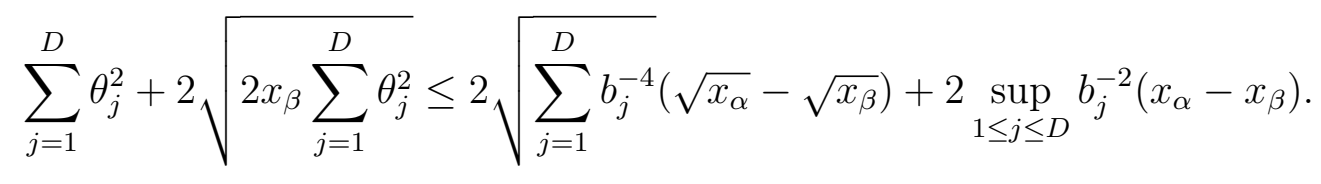

One can check that if

$$
\sum_{j=1}^{D} \theta_{j}^{2} \leq \sqrt{\sum_{j=1}^{D} b_{j}^{-4}}\left(\sqrt{x_{\alpha}}-\sqrt{x_{\beta}}\right)+\sup _{1 \leq j \leq D} b_{j}^{-2}\left(x_{\alpha}-x_{\beta}\right)-x_{\beta}
$$

then (15) holds, and thus $\mathbb{P}_{f}\left(\Phi_{\alpha, D}^{I P}=1\right) \leq \beta$.

For the sake of simplicity, we assume that $b_{j}=j^{-s}$ for mildly ill-posed inverse problems and $b_{j}=\exp (-\gamma j)$ for severely ill-posed inverse problems. We consider the function $f \in H_{D}$ defined by $\theta_{1}^{2}=C \sqrt{D}$ with $C>0$, and $\theta_{j}=0$ for all $j>1$. Then

$$
\|T f\|^{2}=\sum_{j=1}^{D} b_{j}^{2} \theta_{j}^{2}=\sum_{j=1}^{D} \theta_{j}^{2}=C \sqrt{D} .
$$

Moreover, the right hand term in (16) is respectively bounded from below by

$$
C(\alpha, \beta, s) D^{1 / 2+2 s}-x_{\beta}
$$


in the polynomial case and by

$$
C(\alpha, \beta, \gamma) \exp (2 D \gamma)-x_{\beta}
$$

when $b_{j}=\exp (-\gamma j)$.

Hence, for $D$ large enough $(15)$ holds and $\left(\Phi_{\alpha, D}^{I P}, D \geq 1\right)$ is not rate-optimal for $H_{0}^{D P}$ over $\left(K_{D}, D \geq 1\right)$.

Let us now prove the third point of Theorem 4.2. We consider severely ill-posed inverse problems. We introduce the following testing procedure : for all $D \geq 1$, let

$$
\Phi_{\alpha, D}^{D P}=\mathbf{1}_{\left\{\sum_{j=1}^{D} Y_{j}^{2} \geq \bar{s}_{D, \alpha}\right\}} \text { with } \bar{s}_{D, \alpha}=D+2 \sqrt{D x_{\alpha}}+2 x_{\alpha} .
$$

It is proved in [2] that the collection of level- $\alpha$ tests $\left(\Phi_{\alpha, D}^{D P}, D \geq 1\right)$ is rate-optimal for $H_{0}^{D P}$ on $\left(K_{D}, D \geq 1\right)$. For all $C>0$, we want to find $D \geq 1$ and $T f \in K_{D}$ such that $\|f\|^{2} \geq C\left(\sum_{j=}^{D} b_{j}^{-4}\right)^{1 / 2}$ and

$$
P_{f}\left(\Phi_{\alpha, D}^{D P}=1\right) \leq \beta
$$

Let $q_{1-\beta}^{\prime}(D)$ denote the $(1-\beta)$ quantile of $\sum_{j=1}^{D} Y_{j}^{2}$. If $\bar{s}_{D, \alpha} \geq q_{1-\beta}^{\prime}(f)$, we have $\mathbb{P}_{f}\left(\Phi_{\alpha, D}^{D P}=\right.$ $1) \leq \beta$. Using (11) with $\sigma=1$, the condition $\bar{s}_{D, \alpha} \geq q_{1-\beta}^{\prime}(D)$ is satisfied if

$$
\sum_{j=1}^{D} b_{j}^{2} \theta_{j}^{2}+2 \sqrt{\Sigma^{\prime} x_{\beta}} \leq 2 \sqrt{D x_{\alpha}}+2\left(x_{\alpha}-x_{\beta}\right) .
$$

By similar computations as for the second point, this condition holds if

$$
\sum_{j=1}^{D} b_{j}^{2} \theta_{j}^{2}+2 \sqrt{2 x_{\beta} \sum_{j=1}^{D} b_{j}^{2} \theta_{j}^{2}} \leq 2 \sqrt{D}\left(\sqrt{x_{\alpha}}-\sqrt{x_{\beta}}\right)+2\left(x_{\alpha}-x_{\beta}\right) .
$$

We consider the function $f \in H_{D}$ defined by $\theta_{D}=C b_{D}^{-1}$ with $C>0$, and $\theta_{j}=0$ for $j \neq D$. Then

$$
\sum_{j=1}^{D} b_{j}^{2} \theta_{j}^{2}=C
$$

Hence (17) holds for $D$ large enough.

\subsection{Proof of Lemma 3.2}

Assume that

$$
f \in\left\{\nu \in \mathcal{E}_{a, 2}^{\mathcal{X}}(R),\|\nu\|^{2} \geq \gamma_{\sigma}\right\}
$$


Then, for all $m \geq 1$

$$
\|T f\|^{2}=\sum_{k \geq 1} b_{k}^{2} \theta_{k}^{2} \geq \sum_{k=1}^{m} b_{k}^{2} \theta_{k}^{2} \geq b_{m}^{2} \sum_{k=1}^{m} \theta_{k}^{2}=b_{m}^{2}\left(\|f\|^{2}-\sum_{k>m} \theta_{k}^{2}\right) .
$$

Since $f \in \mathcal{E}_{a, 2}^{\mathcal{X}}(R)$

$$
\sum_{k>m} \theta_{k}^{2} \leq a_{m}^{-2} \sum_{k>m} a_{k}^{2} \theta_{k}^{2} \leq R^{2} a_{m}^{-2}
$$

Hence

$$
\|T f\|^{2} \geq b_{m}^{2}\left(\gamma_{\sigma}-R^{2} a_{m}^{-2}\right) .
$$

We conclude the proof choosing $m=m(\sigma)$ such that $R^{2} a_{m(\sigma)}^{-2} \leq c \gamma_{\sigma}$, for some $0<c<1$ independent of $\sigma$.

\subsection{Proof of Theorem 3.1}

Let $\alpha$ and $\beta$ be fixed. Denote by $\rho^{2}\left(\mathcal{E}_{a, 2}^{\mathcal{X}}(R), \alpha, \beta\right)$ and $\rho^{2}\left(\mathcal{E}_{c, 2}^{\mathcal{Y}}(R), \alpha, \beta\right)$ the minimax rates of testing on respectively $\mathcal{E}_{a, 2}^{\mathcal{X}}(R)$ and $\mathcal{E}_{c, 2}^{\mathcal{Y}}(R)$. Let $\Phi_{\alpha}$ a level- $\alpha$ test minimax for $H_{0}^{D P}$ on $\mathcal{E}_{c, 2}^{\mathcal{Y}}(R)$. Then there exists $C_{1}$ positive constant such that

$$
\sup _{T f \in \mathcal{E}_{c, 2}^{\mathcal{Y}}(R),\|T f\|^{2} \geq C_{1} \rho^{2}\left(\mathcal{E}_{c, 2}^{\mathcal{Y}}(R), \alpha, \beta\right)} P_{\theta}\left(\Phi_{\alpha}=0\right) \leq \beta .
$$

Let $C_{2}>0$, thanks to Lemma 3.2 with $\gamma_{\sigma}=C_{2} \rho^{2}\left(\mathcal{E}_{a, 2}^{\mathcal{X}}(R), \alpha, \beta\right)$

$$
\begin{aligned}
& \left\{f \in \mathcal{E}_{a, 2}^{\mathcal{X}}(R),\|f\|^{2} \geq C_{2} \rho^{2}\left(\mathcal{E}_{a, 2}^{\mathcal{X}}(R), \alpha, \beta\right)\right\} \\
& \quad \subset\left\{f, T f \in \mathcal{E}_{c, 2}^{\mathcal{Y}}(R),\|T f\|^{2} \geq(1-c) C_{2} b_{m(\sigma)}^{2} \rho^{2}\left(\mathcal{E}_{a, 2}^{\mathcal{X}}(R), \alpha, \beta\right)\right\} .
\end{aligned}
$$

Hence, $\Phi_{\alpha}$ is a level- $\alpha$ test minimax for $H_{0}^{I P}$ as soon as

$$
R^{2} a_{m(\sigma)}^{-2} \leq c C_{2} \rho^{2}\left(\mathcal{E}_{a, 2}^{\mathcal{X}}(R), \alpha, \beta\right)
$$

for some $0<c<1$ and

$$
C_{1} \rho^{2}\left(\mathcal{E}_{c, 2}^{\mathcal{Y}}(R), \alpha, \beta\right) \leq(1-c) C_{2} b_{m(\sigma)}^{2} \rho^{2}\left(\mathcal{E}_{a, 2}^{\mathcal{X}}(R), \alpha, \beta\right) .
$$

We verify that these both inequalities hold for the four different cases given by the two degrees of ill-posedness of the operator and the two different sets of smoothness conditions displayed in Table 1 . To this end, we use the minimax rates of testing established in [2] and [10].

In the following we write $\mu(\sigma) \simeq \nu(\sigma)$ if there exists two constants $c_{1}$ and $c_{2}$ such 
that for all $\sigma>0, c_{1} \leq \mu(\sigma) / \nu(\sigma) \leq c_{2}$. For all $x \geq 0$, we denote by $\lfloor x\rfloor$ the greatest integer smaller than $x$ and by $\lceil x\rceil$ the smallest integer greater than $x$. Without loss of generality, we assume that $0<\sigma<\sigma_{1}$ for some $0<\sigma_{1}<1$.

1st case: Assume that $\left(a_{k}\right)_{k \geq 1} \sim\left(k^{s}\right)_{k \geq 1}$ for some $s>0$ and that the problem is mildly ill-posed: $\left(b_{k}\right)_{k \geq 1} \sim\left(k^{-t}\right)_{k \geq 1}$. In this setting, recall that

$$
\rho^{2}\left(\mathcal{E}_{a, 2}^{\mathcal{X}}(R), \alpha, \beta\right) \simeq \sigma^{\frac{4 s}{2 s+2 t+1 / 2}} \text { and } \rho^{2}\left(\mathcal{E}_{c, 2}^{\mathcal{Y}}(R), \alpha, \beta\right) \simeq \sigma^{\frac{4 s+4 t}{2 s+2 t+1 / 2}}
$$

We define

$$
m(\sigma)=\left\lceil\sigma^{\frac{-2}{2 s+2 t+1 / 2}}\right\rceil
$$

Then

$$
R^{2} a_{m(\sigma)}^{-2} \simeq m(\sigma)^{-2 s} \simeq \sigma^{\frac{4 s}{2 s+2 t+1 / 2}} \leq c C_{2} \rho^{2}\left(\mathcal{E}_{a, 2}^{\mathcal{X}}(R), \alpha, \beta\right)
$$

where $C_{2}>0$ and $0<c<1$. Moreover

$$
b_{m(\sigma)}^{2} \rho^{2}\left(\mathcal{E}_{a, 2}^{\mathcal{X}}(R), \alpha, \beta\right) \simeq m(\sigma)^{-2 t} \rho^{2}\left(\mathcal{E}_{a, 2}^{\mathcal{X}}(R), \alpha, \beta\right) \simeq \sigma^{\frac{4 t+4 s}{2 s+2 t+1 / 2}} \simeq \rho^{2}\left(\mathcal{E}_{c, 2}^{\mathcal{Y}}(R), \alpha, \beta\right) .
$$

2nd case: Assume that $\left(a_{k}\right)_{k \geq 1} \sim\left(\exp \left(\nu k^{s}\right)\right)_{k \geq 1}$ for some $\nu, s>0$ and that the problem is mildly ill-posed : $\left(b_{k}\right)_{k \geq 1} \sim\left(k^{-t}\right)_{k \geq 1}$. In this setting, remark that the sequence $\left(a_{k} b_{k}^{-1}\right)_{k \in \mathbb{N}}$ satisfies the inequality $\tilde{c_{1}} e^{\nu k^{s}} \leq a_{k} b_{k}^{-1} \leq \tilde{c_{2}} e^{2 \nu k^{s}}$ for all $k \in \mathbb{N}^{\star}$. Hence, using Lemma 7.3

$$
\rho^{2}\left(\mathcal{E}_{a, 2}^{\mathcal{X}}(R), \alpha, \beta\right) \simeq \sigma^{2}\left(\log \left(\sigma^{-2}\right)\right)^{\frac{2 t+1 / 2}{s}} \text { and } \rho^{2}\left(\mathcal{E}_{c, 2}^{\mathcal{Y}}(R), \alpha, \beta\right) \simeq \sigma^{2}\left(\log \left(\sigma^{-2}\right)\right)^{1 / 2 s} .
$$

We define

$$
m(\sigma)=\left\lceil\left(\frac{1}{2 \nu} \ln \left(\sigma^{-2}\right)\right)^{1 / s}\right\rceil .
$$

Then, for $\sigma$ small enough, $m(\sigma) \geq 1$ and

$$
R^{2} a_{m(\sigma)}^{-2} \simeq R^{2} \sigma^{2} \leq R^{2} \sigma^{2}\left(\ln \left(\sigma^{-2}\right)\right)^{(2 t+1 / 2) / s} \leq c C_{2} \rho^{2}\left(\mathcal{E}_{a, 2}^{\mathcal{X}}(R), \alpha, \beta\right),
$$

where $C_{2}>0$ and $0<c<1$. Moreover

$$
b_{m(\sigma)}^{2} \rho^{2}\left(\mathcal{E}_{a, 2}^{\mathcal{X}}(R), \alpha, \beta\right) \simeq \sigma^{2}\left(\ln \sigma^{-2}\right)^{1 / 2 s} \simeq \rho^{2}\left(\mathcal{E}_{c, 2}^{\mathcal{Y}}(R), \alpha, \beta\right) .
$$

3rd case: Assume that $\left(a_{k}\right)_{k \in \mathbb{N}} \sim\left(k^{s}\right)_{k \geq 1}$ for some $s>0$ and that the problem is severely ill-posed: $\left(b_{k}\right)_{k \geq 1} \sim\left(e^{-\gamma k}\right)_{k \geq 1}$ for some $\gamma>0$. In this setting, remark that the sequence $c=\left(c_{k}\right)_{k \geq 1}=\left(a_{k} b_{k}^{-1}\right)_{k \in \mathbb{N}}$ satisfies the inequality $\tilde{c_{1}} e^{\nu k} \leq c_{k} \leq \tilde{c_{2}} e^{2 \nu k}$ for all $k \in \mathbb{N}^{\star}$. Hence, using Lemma 7.3

$$
\rho^{2}\left(\mathcal{E}_{a, 2}^{\mathcal{X}}(R), \alpha, \beta\right) \simeq\left(\log \left(\sigma^{-2}\right)\right)^{-2 s} \text { and } \rho^{2}\left(\mathcal{E}_{c, 2}^{\mathcal{Y}}(R), \alpha, \beta\right) \simeq \sigma^{2}\left(\log \left(\sigma^{-2}\right)\right)^{1 / 2}
$$


We set

$$
m(\sigma)=\left\lfloor\frac{1}{2 \gamma} \log \left(\sigma^{-2} \log \left(\sigma^{-2}\right)^{-1 / 2}\right)+\frac{1}{2 \gamma} \log \left(\log \left(\sigma^{-2}\right)^{-2 s}\right)\right\rfloor .
$$

Then

$$
R^{2} a_{m(\sigma)}^{-2} \leq C R^{2} m(\sigma)^{-2 s} \leq C\left(\log \left(\sigma^{-2}\right)\right)^{-2 s} \leq c C_{2} \rho^{2}\left(\mathcal{E}_{a, 2}^{\mathcal{X}}(R), \alpha, \beta\right),
$$

where $C_{2}>0,0<c<1$, and

$$
b_{m(\sigma)}^{2} \rho^{2}\left(\mathcal{E}_{a, 2}^{\mathcal{X}}(R), \alpha, \beta\right) \geq C e^{-2 \gamma m(\sigma)} \log \left(\sigma^{-2}\right)^{-2 s} \geq C \sigma^{2}\left(\log \left(\sigma^{-2}\right)\right)^{1 / 2} \simeq \rho^{2}\left(\mathcal{E}_{c, 2}^{\mathcal{Y}}(R), \alpha, \beta\right)
$$

4th case: Assume that $\left(a_{k}\right)_{k \geq 1} \sim\left(\exp \left(\nu k^{s}\right)\right)_{k \geq 1}$ for some $\nu, s>0$ and that the problem is severely ill-posed: $\left(b_{k}\right)_{k \geq 1} \sim\left(e^{-\gamma k}\right)_{k \geq 1}$ for some $\gamma>0$. In this setting, remark that the sequence $c=\left(c_{k}\right)_{k \geq 1}=\left(a_{k} b_{k}^{-1}\right)_{k \in \mathbb{N}}$ satisfies the inequality $\tilde{c_{1}} e^{\gamma k} \leq c_{k} \leq \tilde{c_{2}} e^{2 \gamma k}$ for all $k \in \mathbb{N}^{\star}$. Hence, using Lemma 7.3

$$
\rho^{2}\left(\mathcal{E}_{a, 2}^{\mathcal{X}}(R), \alpha, \beta\right) \simeq e^{-2 \nu \tilde{D}^{s}} \text { and } \rho^{2}\left(\mathcal{E}_{c, 2}^{\mathcal{Y}}(R), \alpha, \beta\right) \simeq \sigma^{2}\left(\log \left(\sigma^{-2}\right)\right)^{1 / 2},
$$

where $\tilde{D}$ denotes the integer part of the solution of $2 \nu D^{s}+2 \gamma D=\log \left(\sigma^{-2}\right)$. Then, we set

$$
m(\sigma)=\left\lfloor\frac{1}{2 \gamma} \log \left(\sigma^{-2} \log \left(\sigma^{-2}\right)^{1 / 2}\right)-\frac{\nu}{\gamma} \tilde{D}^{s}\right\rfloor .
$$

Remark that for $\sigma$ small enough, $m(\sigma) \geq \tilde{D}$ since $s<1$. Therefore

$$
R^{2} a_{m(\sigma)}^{-2}=R^{2} e^{-2 \nu m(\sigma)^{s}} \leq c C_{2} e^{-2 \nu \tilde{D}^{s}},
$$

where $C_{2}>0$ and $0<c<1$. Then

$$
b_{m(\sigma)}^{2} \rho^{2}\left(\mathcal{E}_{a, 2}^{\mathcal{X}}(R), \alpha, \beta\right) \simeq e^{-2 \gamma m(\sigma)-2 \nu \tilde{D}^{s}} \geq C \sigma^{2}\left(\log \left(\sigma^{-2}\right)\right)^{1 / 2} .
$$

This concludes the first part of Theorem 3.1. In the second part, we have to find a level- $\alpha$ test minimax for $H_{0}^{I P}$ on $\mathcal{E}_{a, 2}^{\mathcal{X}}(R)$ but not for $H_{0}^{D P}$ on $\mathcal{E}_{c, 2}^{\mathcal{Y}}(R)$. For the sake of convenience, we assume that the problem is mildly ill-posed. The proof follows essentially the same lines when $\left(b_{k}\right)_{k \geq 1}$ is an exponentially decreasing sequence. Recall that

$$
\rho^{2}\left(\mathcal{E}_{a, 2}^{\mathcal{X}}(R), \alpha, \beta\right)=\sup _{D \in \mathbb{N}^{*}}\left(\rho_{D}^{2} \wedge R^{2} a_{D}^{-2}\right), \text { with } \rho_{D}^{2} \simeq \sigma^{2} D^{2 s+1}
$$

and

$$
\rho^{2}\left(\mathcal{E}_{c, 2}^{\mathcal{Y}}(R), \alpha, \beta\right)=\sup _{D \in \mathbb{N}^{*}}\left(\tilde{\rho}_{D}^{2} \wedge R^{2} b_{D}^{2} a_{D}^{-2}\right), \text { with } \tilde{\rho}_{D}^{2} \simeq \sigma^{2} \sqrt{D}
$$


Hence, we are looking for a function $f$ and a level- $\alpha$ test $\Phi_{\alpha} \operatorname{minimax}$ for $H_{0}^{I P}$ on $\mathcal{E}_{a, 2}^{\mathcal{X}}(R)$ verifying:

$$
T f \in \mathcal{E}_{c, 2}^{\mathcal{Y}}(R),\|T f\|^{2} \geq C_{\alpha, \beta} \sup _{D \in \mathbb{N}^{*}}\left(\tilde{\rho}_{D}^{2} \wedge R b_{D}^{2} a_{D}^{-2}\right) \text { and } P_{f}\left(\Phi_{\alpha}=0\right)>\beta .
$$

To this end, introduce

$$
D^{\star}=\inf \left\{D: \tilde{\rho}_{D}^{2} \geq R^{2} b_{D}^{2} a_{D}^{-2}\right\}
$$

Clearly

$$
\|T f\|^{2} \geq C_{\alpha, \beta} \tilde{\rho}_{D^{\star}}^{2} \Rightarrow\|T f\|^{2} \geq C_{\alpha, \beta} \sup _{D \in \mathbb{N}^{*}}\left(\tilde{\rho}_{D}^{2} \wedge R b_{D}^{2} a_{D}^{-2}\right) .
$$

Let $f$ the function defined as

$$
\theta_{1}^{2}=C_{\alpha, \beta} \sigma^{2} \sqrt{D^{\star}} \text { and } \theta_{j}=0 \forall j>1 .
$$

The function $f$ belongs to the space $H_{D^{\star}}$ and $\|T f\|^{2} \geq C_{\alpha, \beta} \sigma^{2} \sqrt{D^{\star}}$. Moreover, $T f \in$ $\mathcal{E}_{c, 2}^{\mathcal{Y}}(R)$ since

$$
\sum_{k=1}^{+\infty} a_{k}^{2} b_{k}^{-2}\left\langle T f, \psi_{k}\right\rangle^{2}=a_{1}^{2} \theta_{1}^{2} \simeq C_{\alpha, \beta} \sigma^{2} \sqrt{D^{\star}} \leq Q,
$$

at least for $\sigma$ small enough. Moreover, from Theorem 4.2, we deduce that the global test $\Phi_{D^{\star}, \alpha}$ defined in (12)-(13) is not powerful for $H_{0}^{D P}$ when the alternative is defined by (18).

\section{References}

[1] F. Abramovich and B. W. Silverman. Wavelet decomposition approaches to statistical inverse problems. Biometrika, 85(1):115-129, 1998.

[2] Yannick Baraud. Non-asymptotic minimax rates of testing in signal detection. Bernoulli, 8:577-606, 2002.

[3] N. Bissantz, G. Claeskens, H. Holzmann, and A. Munk. Testing for lack of fit in inverse regression, with applications to biophotonic imaging. preprint, 2008.

[4] Cristina Butucea, Catherine Matias, and Christophe Pouet. Adaptive goodnessof-fit testing from indirect observations. Ann. Inst. Henri Poincaré Probab. Stat., 45(2):352-372, 2009.

[5] L. Cavalier and N.W. Hengartner. Adaptative estimation for inverse problems with noisy operators. Inverse Problems, 21:1345-1361, 2005.

[6] David L. Donoho. Nonlinear solution of linear inverse problems by wavelet-vaguelette decomposition. Appl. Comput. Harmon. Anal., 2(2):101-126, 1995. 
[7] Hajo Holzmann, Nicolai Bissantz, and Axel Munk. Density testing in a contaminated sample. J. Multivariate Anal., 98(1):57-75, 2007.

[8] Sapatinas T. Ingster, Yu.I. and I.A. Suslina. Minimax signal detection in ill-posed inverse problems. 2010. Working paper.

[9] Yu. I. Ingster. Asymptotically minimax hypothesis testing for nonparametric alternatives. I. Math. Methods Statist., 2(2):85-114, 1993.

[10] B. Laurent, J-M. Loubes, and C. Marteau. Non asymptotic minimax rates of testing in signal detection with heterogeneous variances. ArXIv: 0912.2423.

[11] Oleg V. Lepski and Vladimir G. Spokoiny. Minimax nonparametric hypothesis testing: the case of an inhomogeneous alternative. Bernoulli, 5(2):333-358, 1999.

[12] Jean-Michel Loubes and Carenne Ludeña. Model selection for non linear inverse problems. ESAIM PS.

[13] Jean-Michel Loubes and Carenne Ludeña. Adaptive complexity regularization for linear inverse problems. Electron. J. Stat., 2:661-677, 2008. 